\title{
A Metamodel for Collaboration Formalization
}

\author{
Loïc Bidoux ${ }^{1,2}$, Frédérick Bénaben ${ }^{1}$, and Jean-Paul Pignon ${ }^{2}$ \\ ${ }^{1}$ Mines Albi - Université de Toulouse \\ \{loic.bidoux, frederick. benaben\} @mines-albi.fr \\ ${ }^{2}$ Customer Innovation Centre, \\ Thales Communications \& Security \\ \{loic.bidoux, jean-paul.pignon\} athalesgroup.com
}

\begin{abstract}
This paper presents a metamodel formalizing collaboration of heterogeneous entities (organizations, agencies, companies, intelligent agents...). Initially developed to support crisis management, this metamodel has been extended to any objective driven work of collaborative networks $(\mathrm{CN})$. This formalism embeds all concepts (and inter-relations between them) required to represent any collaborative situation aiming at meeting some CN's objectives using sets of capabilities provided by each involved stakeholder. This metamodel is the keystone of a model driven decision support system (under development) designing collaborative processes that can optimize the coordination of collaborative stakeholders. The metamodel expressiveness is briefly illustrated through a use-case.
\end{abstract}

Keywords: knowledge representation, collaboration modeling, metamodel, heterogeneous collaborative networks, crisis management.

\section{Introduction}

As a result of evolving security market needs, new societal challenges and advances in the information and communication technologies, collaboration of organizations has been significantly transformed in the last decades. In order to efficiently seize the various opportunities offered to them, entities structure themselves as collaborative networks (CN) [3] which may be composed of organizations, agencies, companies...

Those CNs can achieve objectives that no individual entity of the network can achieve on its own. Nevertheless, due to the systems of systems nature of this type of structure, new challenges have to be overcome. Indeed, involved stakeholders are often heterogeneous (at cultural, functional and technological levels) and poorly, if at all, trained to work together. This inevitably leads to critical collaboration issues (poor data management and partners' coordination for instance) that may strongly limit the efficiency of the CN's actions. In fact, it is generally quite complex to fill the gap between the willingness to collaborate to seize an opportunity and the design of an effective collaborative process to reach the targeted objectives.

The research project described in [2] aims to produce a system designing semiautomatically such collaborative processes through a model driven approach. In order to realize this system, it is necessary to (i) formalize the entire problem in such a way 
that it can be easily modelled by the CN's members and be processed by a computer at the same time. Then, it became possible to (ii) reason over this knowledge to design processes to be executed by the $\mathrm{CN}$ to reach its goals. The work presented here focuses on the knowledge representation formalism required to model a collaboration problem and its solutions.

The organization of this article is the following: first, a literature review highlights concepts and methods used to represent knowledge as well as related works. In the third section, a collaboration metamodel is proposed. Its expressiveness is illustrated with a use-case in the following section. Next, a concluding section suggests some perspectives for this study.

\section{$2 \quad$ Literature Review}

\subsection{Knowledge Representation and Reasoning}

Metamodelling is the keystone of knowledge representation. A metamodel is a set of concepts and rules referring to those concepts used to build models. In this work, five models are designed over the proposed metamodel: three of them depict the collaboration problem (collaborative situation, objectives to achieve and partner's capabilities models) and the two others refer to its solutions (collaborative processes and evaluation models).

Moreover, a metamodel can be intrinsically considerated as a model of a domain of interest. Therefore, it describes an ontology defined as "an explicit specification of a conceptualization" [5]. Consequently, it means that instances of the five previously models can be injected into an ontology thus building a knowledge base for collaboration of heterogeneous entities. Ultimately, a system such as the one mentioned in section 1 can reason on this knowledge base.

\subsection{Related Work and Contribution beyond the State of the Art}

Two metamodels respectively dedicated to enterprise collaboration [10] and collaborative crisis management [12] have been realized in some previous works. A collaborative metamodel (CMM) generalizing these two studies has also been proposed [8]. The metamodel presented in this article is based on this CMM and uses many of its fundamental ideas.

Main contribution of this work is taking into account new concepts leading to a metamodel with more expressive power than existing ones. Involved concepts are resources and emergent behaviours, feedbacks from past collaborations and multiple criteria decision analysis core notions [1]. Moreover, amongst all existing formalisms representing actions and their effects (see [11] for a comprehensive review), dynamic logic (DL) [6] has been adopted. Even if it is outside the scope of this paper, it is worth mentioning that as a modal logic, DL can infer more expressive statements than those used in previous works when reasoning on the collaboration's ontology. 


\section{Collaboration Metamodel}

\subsection{Metamodel Proposition}

To be as generic and reusable as possible, the proposed metamodel is structured following two dimensions: layers and modules (see figure 1). One can conceptualize layers as a vertical dimension with a root layer describing relevant concepts for any form of collaboration that can be extended through domain specific layers. Modules are much more like a horizontal dimension with a core module encompassing the concepts required to describe the collaboration essence that can be extended by functional modules focusing on specific aspects of a given collaboration.

Thanks to this architecture's flexibility, one can formalize broad collaboration needs by choosing relevant modules and layers. Moreover, it is possible to extend existing modules and layers or to create new ones. The rest of this paper focuses on the root layer description. See [8] for an extension of the root layer into a domain specific layer for road crisis management.

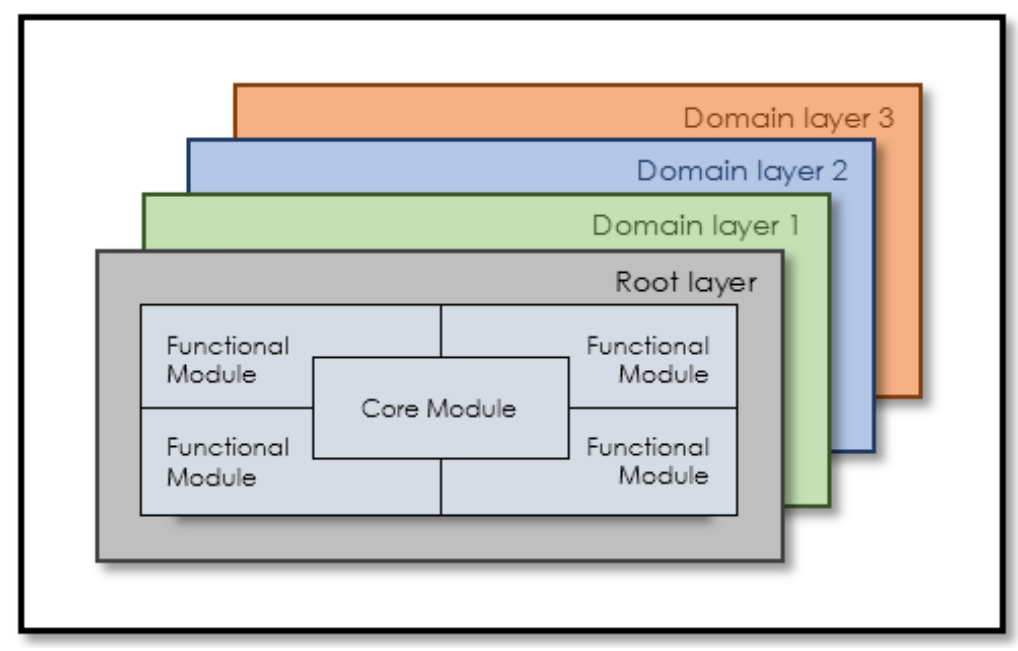

Fig. 1. Metamodel architecture

Figure 2 represents six modules of the root layer. Core, situation representation and process modules embed the main concepts of the metamodel as they respectively formalize the $\mathrm{CN}$ and its objectives, the collaborative situation and the CN's capabilities to execute in order to achieve targeted objectives from the initial situation. They are completed by the evaluation module describing required concepts to assess process module elements. Experience feedback and emergent capability modules are considerably more specific modules: one formalizes the CN's emergent behaviour and the other represents experience to be capitalized to improve future collaborations. 


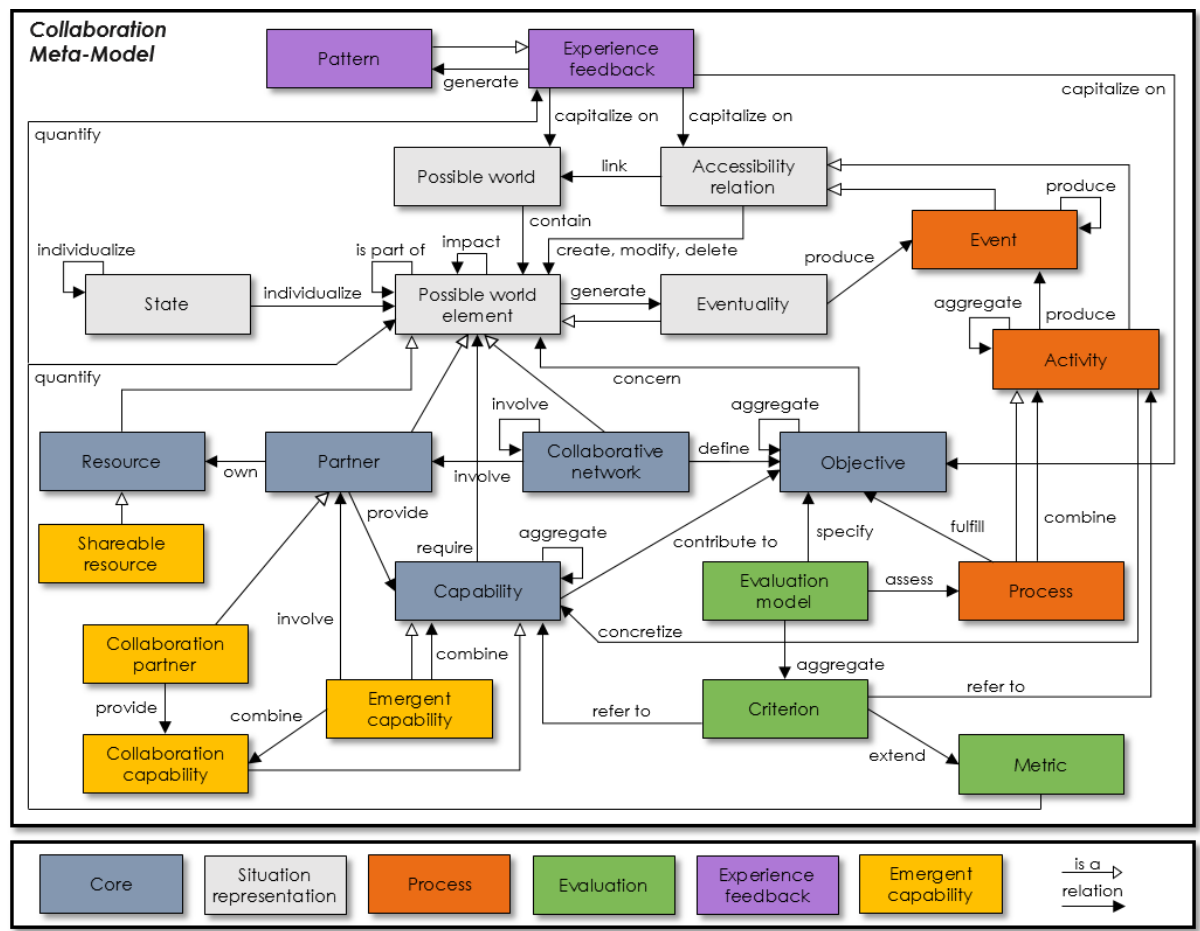

Fig. 2. Collaboration metamodel

\subsection{Metamodel Glossary (sorted by module)}

Capability. Both partner's ability to create, transform or delete possible world elements and availability of the resources required to concretize this ability

Collaborative Network. A set of partners collaborating to achieve an objective

Objective. Effect that a collaborative network wants to produce on a possible world

Partner. Stakeholder of a collaborative network (organization, agency, company, other collaborative network...)

Resource. Possible world element sizing the capability concretization of a partner

Accessibility Relation. Activity or event transforming a possible world into another one

Eventuality. Opportunity or threat that might impact possible world elements 
Possible World. "The maximally inclusive situation encompassing all others: things, as a whole" [9]

Possible World Element. Element constituting a possible world

State. Characteristic individualizing a subset of a possible world element

Activity. Capability concretization

Event. Fact impacting possible world elements when it occurs

Process. Series of activities. Is collaborative if activities are performed by several partners

Criterion. Preference relation on a metric interpretable as a degree of satisfaction

Evaluation Model. Criteria hierarchy and its associated aggregation functions providing a score to a solution, so supporting decision-making over a set of alternatives

Metric. Variable which has a qualitative relation over its domain of definition

Experience Feedback. Capitalization of elements characterizing a collaboration

Pattern. Best practice stemming from experience feedback

Collaboration Capability. Capability of a collaboration partner

Collaboration Partner. Partner (potentially virtual) supporting the coordination of other partners

Emergent Capability. Collective capability emerging from the collaboration of several partners

Shareable Resource. Resource possibly usable by different partners, whose management by the collaborative network can be relevant according to its granularity

\subsection{Metamodel Description}

Core Module. Formalizes concepts required to define a collaboration: some partners providing capabilities and resources group together to form a collaborative network targeting objectives. Note the aggregate relation over capability allowing partners to expose their capabilities with the granularity of their choice. 
Situation Representation Module. Encompasses concepts describing situations using the dynamic logic formalism. Possible worlds depict a static viewpoint of a situation using possible world elements that may be characterized through various states. Eventualities are generated by possible world elements and may impact the world if they occur. Accessibility relation brings a dynamic viewpoint to the model formalizing transformations of a static viewpoint (a possible world) into another one.

The paradigm underlying the design of this module is provided by the dynamic logic. In DL, a directed graph (called a Kripke frame) is generally used to describe situations. Each node of this graph (possible world) is characterized by the truth value of a set of propositional variables (formalized through possible world element). An edge (or accessibility relation) from a node $n 1$ to a node $n 2$ indicates that executing some defined actions in $n 1$ possibly or necessarily leads to the possible world $n 2$.

Process Module. Describes the collaborative processes that the CN may execute to achieve its objectives. A process is a series of activities that can transform a possible world into another one. Moreover, activities may produce some events impacting a possible world.

Evaluation Module. Formalizes concepts required to assess collaborative processes. Some metrics built upon various measures quantify the process aspects that decisionmakers expect to evaluate. A criterion is formed by coupling a utility function and a metric. Such functions indicate the degree of satisfaction associated to each metric value. Finally, the evaluation model is defined by combining together many criteria using an aggregation function.

Experience Feedback Module. This module describes experience feedback gathering to improve future collaborations. Experience feedbacks are capitalized on the situation description, the decision-makers' objectives and the process executed to achieve these objectives. Some patterns for an efficient collaboration may be generated from them.

Emergent Capability Module. Detail concepts used to formalize the emergent behaviour of the $\mathrm{CN}$. Collaboration partners are special stakeholders whose role (not operational) is devoted to optimize coordination between partners. They provide collaboration capabilities and may be virtual such as an information system managing the $\mathrm{CN}$ 's shareable resources. An emergent capability is a capability involving several partners that combines operational and collaboration capabilities.

\section{$4 \quad$ Illustration}

The metamodel expressiveness is illustrated on a use-case through two models: the collaborative situation (figure 3) and the objectives to achieve (table 1) models. The use-case concerns a chemical company which suffers a massive cyber-attack dealing numerous damages: (i) the reactor's sensors of a plant have been manipulated 
removing its self-regulation feature; (ii) an intrusion on a data-center has leaked some strategic documents; (iii) defamatory content have been added to the company website by exploiting a cross-site-scripting security breach.

The collaborative situation model contains possible world elements required to describe the parts of the company under attack (plant, reactor, sensors, datacenter, data, website) as well as other elements related to the cyber-attack (corporate identity, strategy and market value of the enterprise). Moreover, the various states of these elements are also formalized (reactor's sensors are untrustworthy, some data are leaked, datacenter and website are hacked). States implicitly subset the possible world element they refer to thus allowing reasoning over a specific part of a possible world element if necessary. Eventualities threatening the company (the reactor's explosion, additional data leaks and corporate identity degradation) are also included in the model.

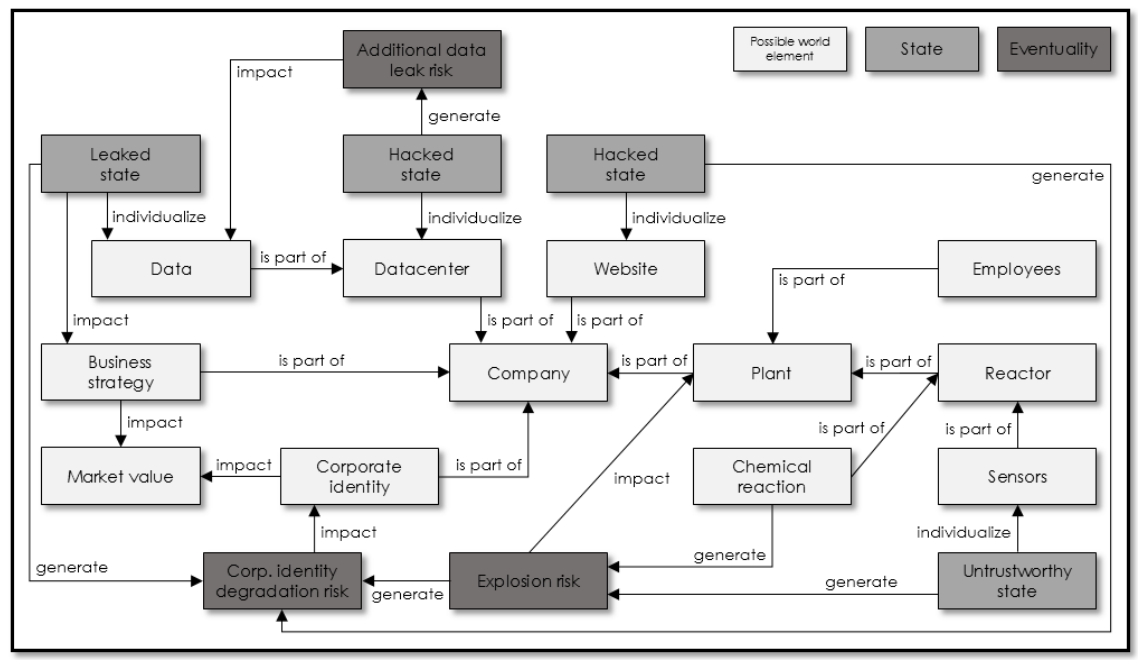

Fig. 3. Collaborative situation model

Decision-makers objectives represent effects the $\mathrm{CN}$ wants to produce on the world, and therefore are formalized through possible world elements.

Table 1. Objectives to achieve ranked by priority

\begin{tabular}{|c|l|}
\hline 1 & Add evacuated state to employees \\
\hline 2 & Remove plant's explosion risk \\
\hline 3 & Remove additional data leak risk \\
\hline 4 & Add off-line state to website \\
\hline 5 & Remove datacenter hacked state \\
\hline
\end{tabular}

\begin{tabular}{|c|l|}
\hline 6 & $\begin{array}{l}\text { Add press release possible world } \\
\text { element }\end{array}$ \\
\hline 7 & $\begin{array}{l}\text { Remove website hacked and off- } \\
\text { line states }\end{array}$ \\
\hline 8 & Remove untrustworthy state \\
\hline
\end{tabular}




\section{$5 \quad$ Conclusion and Future Work}

The metamodel presented throughout this article is dedicated to formalize any collaboration of heterogeneous entities with common goals. Its two-dimensional architecture (based on layers and modules) grants him a great potential of flexibility and reusability. Thanks to the root layer's modules, one can formalize the fundamental aspects of a collaboration (see core, situation representation, process and evaluation modules) as well as more specific ones (refer to experience feedback and emergent capability modules).

Future works will include conception of an axiomatic theory of collaboration based upon this metamodel, using dynamic logic and possibly benefiting from logics for cooperation and preferences [7]. This theory will transform users' inputs gathered through the proposed metamodel into a planning problem. An algorithm coupling automated planning techniques [4] and multiple criteria decision analysis [1] will be defined to design and propose collaborative processes solving this problem.

Acknowledgements. This work is supported by the Customer Innovation Center of Thales Communications \& Security and the French National Association for Research and Technology under number 2012/1334.

\section{References}

1. Belton, V., Stewart, T.: Multiple Criteria Decision Analysis - An Integrated Approach. Springer US (2002)

2. Bidoux, L., Pignon, J.P., Benaben, F.: A model driven system to support optimal collaborative processes design in crisis management. In: Proceedings of the 11th International Conference on Information Systems for Crisis Response and Management (2014)

3. Camarinha-Matos, L.M., Afsarmanesh, H.: Collaborative networks: a new scientific discipline. Journal of Intelligent Manufacturing 16(4-5), 439-452 (2005)

4. Ghallab, M., Nau, D., Traverso, P.: Automated Planning: Theory and Practice. Morgan Kauffmann Publishers (2004)

5. Gruber, T.R.: Toward Principles for the Design of Ontologies Used for Knowledge Sharing. Journal of Human-Computer Studies 43(5-6), 907-928 (1995)

6. Harel, D., Kozen, D., Tiuryn, J.: Dynamic Logic. Foundations of computing. MIT Press (2000)

7. Kurzen, L.: Logics for Cooperation, Actions and Preferences. Master's thesis, Universiteit van Amsterdam (2007)

8. Mace-Ramete, G., Lamothe, J., Lauras, M., Benaben, F.: A road crisis management metamodel for an information decision support system. In: 6th IEEE International Conference on Digital Ecosystems Technologies (2012)

9. Menzel, C.: Possible worlds. In: Zalta, E.N. (ed.) The Stanford Encyclopedia of Philosophy, Spring 2014 edn. (2014) 
10. Rajsiri, V.: Knowledge-based system for collaborative process specification. Ph.D. thesis, Ecole des Mines d'Albi-Carmaux - Universite de Toulouse (2009)

11. Segerberg, K., Meyer, J.J., Kracht, M.: The logic of action. In: Zalta, E.N. (ed.) The Stanford Encyclopedia of Philosophy, winter 2013 edn. (2013)

12. Truptil, S., Benaben, F., Couget, P., Lauras, M., Chapurlat, V., Pingaud, H.: Interoperability of Information Systems in Crisis Management: Crisis Modeling and Metamodeling. In: Enterprise Interoperability III (2008) 